\title{
AVALIAÇÃO DOS POTENCIAIS EVOCADOS AUDITIVOS DO TRONCO ENCEFÁLICO NA ESCLEROSE MÚLTIPLA
}

\author{
Marco Aurélio Rocha Santos', Marco Aurélio Lana Peixoto², \\ Mário Sérgio Lei Munhoz ${ }^{3}$, Alessandra Varella de Almeida ${ }^{4}$
}

\begin{abstract}
RESUMO - O objetivo do presente estudo foi avaliar a incidência de alterações auditivas e dos potenciais evocados auditivos do tronco encefálico (PEATE) em indivíduos portadores de esclerose múltipla (EM). Participaram do estudo 20 indivíduos do sexo feminino e 9 do masculino com diagnóstico definido de EM, sem sinais clínicos ou de alterações à ressonância nuclear magnética de acometimento do tronco encefálico. Testes audiométricos e a pesquisa dos PEATE foram realizados em todos os indivíduos.Utilizou-se da classificação dos PEATE proposta por Jerger (1986) na análise da morfologia das ondas nos portadores de EM. Dos 58 PEATE realizados encontrou-se $55 \%$ classificados como tipo I (resposta normal ) na classificação de Jerger em ambos os sexos. Considerando-se como alterados as avaliações com PEATE dos indivíduos portadores de EM que apresentaram os demais tipos: II, III, IV ou V da classificação de Jerger em pelo menos um dos lados, encontrou-se $60 \%$ de alterações no sexo feminino e $56 \%$ no masculino, totalizando $58,62 \%$. Estes achados enfatizam a relevância do estudo dos PEATE em casos de suspeita clínica de doenças desmielinizantes e naqueles com diagnóstico definido de EM.
\end{abstract}

PALAVRAS-CHAVE: potenciais evocados auditivos, tronco encefálico, esclerose múltipla.

\begin{abstract}
Auditory evoked potentials in multiple sclerosis
ABSTRACT - The aim of this study was to evaluate the incidence of auditory and neurotological disorders in multiple sclerosis (MS). Twenty female and 9 male with a definite diagnosis of multiple sclerosis without signs of involvement of the brain stem underwent an audiological and an early auditory evoked potentials (EAEP). The wave forms were classified according to Jerger's (1986) classification. In 58 EAEP it was found 55\% of type I (normal response) according to Jerger's classification in both sexes. Considering as an abnormal response the EAEP classified in type II, III, IV or V according to Jerger in at least one side, it was found $60 \%$ of abnormalities in females and $56 \%$ in males, totalizing $58,62 \%$ of all the studied subjects. The authors emphasize the use of EAEP in MS.
\end{abstract}

KEY WORDS: auditory evoked potentials, brainstem multiple sclerosis.

Os potenciais evocados representam respostas elétricas do sistema nervoso a um estímulo externo ${ }^{1}$. As respostas auditivas do tronco encefálico, também denominadas potenciais evocados auditivos do tronco encefálico (PEATE), demonstram a atividade do sistema auditivo que é produzida em resposta a um estímulo acústico, sendo geradas no nervo acústico e no tronco encefálico. Os PEATE são caracterizados por uma série de ondas com picos positivos que surgem nos primeiros dez milisegundos após o estímulo, usualmente o clique. Estas ondas representam a somatória da atividade neural de um ou mais sítios geradores dentro do sistema nervoso, sendo as mais importantes as ondas I, III e V, que representam atividade elétrica no nervo auditivo, núcleo coclear e lemnisco lateral / colículo inferior, respectivamente.

As aplicações clínicas dos PEATE são várias: vertigem, acúfenos, perdas auditivas assimétricas, perdas auditivas progressivas, avaliação da sensibilidade auditiva e na avaliação de doenças neurológicas, incluindo lesões tumorais, lesões difusas, doenças desmielinizantes e avaliação de anormalidades das vias auditivas neurais do nervo acústico e do tronco encefálico² ${ }^{2}$.

Disciplina de Otoneurologia - Universidade Federal de São Paulo - Escola Paulista de Medicina, São Paulo SP, Brasil (EPM - UNIFESP) e Centro de Investigação em Esclerose Múltipla Universidade Federal de Minas Gerais, Belo Horizonte MG, Brasil (UFMG): ${ }^{1}$ Doutourando em Otorrinolaringologia EPM-UNIFESP; ${ }^{2}$ Professor Adjunto dos Departamentos de Neurologia e Oftalmologia da UFMG; ${ }^{3}$ Professor Associado Livre Docente da Disciplina de Otoneurologia da EPM-UNIFESP; ${ }^{4}$ Especialista em Distúrbios da Comunicação Humana - EPM-UNIFESP. 
A esclerose múltipla (EM) é o tipo mais comum de doença desmielinizante ${ }^{3}$, sendo predominantemente encontrada em adultos jovens, tendo curso lento e progressivo, com períodos de exarcebação e remissão dos sintomas específicos. São freqüentes alterações no nervo óptico, medula espinhal, tronco encefálico e regiões periventriculares. $O$ diagnóstico é clínico ${ }^{4}$, baseado no envolvimento múltiplo do sistema nervoso, com auxílio da pesquisa de bandas oligoclonais e imunoglobulinas no líquido cefalorraquidiano, tomografia computadorizada, ressonância nuclear magnética, potenciais evocados visuais, somatosensitivos e auditivos. A realização dos PEATE em doenças desmielinizantes é útil em indivíduos com envolvimento do sistema nervoso central. Alterações nos PEATE sugerem a presença de lesão, não suspeitada clinicamente ${ }^{1}$. A incidência de alterações nos PEATE nos indivíduos portadores de EM é variável na literatura, sendo que as mais comumente encontradas são o prolongamento dos intervalos interpicos, ausência de ondas e pouca reprodutibilidade ${ }^{2}$.

O objetivo do presente estudo foi avaliar a incidência de alterações auditivas dos PEATE em indivíduos portadores de esclerose múltipla, avaliando as alterações nas latências absolutas, intervalos interpicos, características das ondas, morfologia, replicabilidade das ondas, utilizando-se da classificação de Jerger ${ }^{5}$.

\section{MÉTODO}

Participaram do estudo 20 indivíduos do sexo feminino e 9 do masculino com diagnóstico de EM. As idades do grupo feminino foram de 20 a 61 anos, com média de 43,8 anos e do masculino variaram de 26 a 50 anos, com média de 38,22 anos, provenientes do Centro de Investigação em Esclerose Múltipla de Minas Gerais - Universidade Federal de Minas Gerais que apresentavam diagnóstico definitivo de EM, sem sinais clínicos ou de alterações à ressonância nuclear magnética de acometimento do tronco encefálico.

Os indivíduos foram submetidos à avaliação neurológica, otorrinolaringológica e audiológica constando de: anamnese e exame clínico otorrinolaringológico, testes audiométricos através de audiometria tonal e vocal incluíndo índice de reconhecimento de fala e imitanciometria com pesquisa de reflexo estapediano.

Os testes audiométricos foram realizados com audiômetro Madsen Midimate $622^{\circledR}$ em cabine acusticamente tratada e seguiram os moldes propostos por Mangabeira Albernaz ${ }^{6}$. Os testes imitanciométricos foram feitos com impedanciômetro Amplaid $750^{\circledR}$.

Foram seguidos os critérios propostos por Davis e Silver$\operatorname{man}^{7}$ na classificação das audiometrias e por Russo ${ }^{8}$ na classificação das imitanciometrias.

A pesquisa dos PEATE foi realizada em todos os indivíduos utilizando equipamento Amplaid MK $12^{\circledR}$ de quatro canais, em sala com ambiente silencioso, sem tratamento acústico, em decúbito dorsal, sem utilização de sedação.

Inicialmente foi realizada pesquisa para detecção do limiar psicoacústico para o clique em cada indivíduo. Em seguida foi realizada a colocação de eletrodos de superfí-

Tabela 1. Formas clínicas segundo a classificação de Lublin e Reingold (1996).

\begin{tabular}{lcccc}
\hline & \multicolumn{2}{c}{ Feminino } & \multicolumn{2}{c}{ Masculino } \\
\cline { 2 - 5 } Forma clínica & $\mathrm{N}^{\circ}$ & $\%$ & $\mathrm{~N}^{\circ}$ & $\%$ \\
\hline Progressiva Primária & 3 & 15 & 1 & 11,11 \\
Progressiva Secundária & 2 & 10 & 0 & 0 \\
Surto Progressiva & 5 & 25 & 1 & 11,11 \\
Surto Remissiva & 10 & 50 & 7 & 77,77 \\
Total & 20 & 100 & 9 & 100 \\
\hline
\end{tabular}

Tabela 2. Classificação das perdas auditivas segundo Davis e Silverman (1970).

\begin{tabular}{lccccccccc}
\hline & \multicolumn{4}{c}{ Sexo feminino } & \multicolumn{3}{c}{ Sexo masculino } \\
\cline { 2 - 9 } & \multicolumn{2}{c}{ OD } & \multicolumn{2}{c}{ OE } & OD & \multicolumn{2}{c}{ OE } \\
\cline { 2 - 9 } Classificação & $\mathrm{n}^{\circ}$ & $\%$ & $\mathrm{n}^{\circ}$ & $\%$ & $\mathrm{n}^{\circ}$ & $\%$ & $\mathrm{n}^{\circ}$ & $\%$ \\
\hline Normal & 18 & 90 & 14 & 70 & 7 & 77,7 & 7 & 77,7 \\
Leve & 1 & 5 & 4 & 20 & 1 & 11,1 & 0 & 0 \\
Moderada & 1 & 5 & 2 & 10 & 1 & 11,1 & 1 & 11,1 \\
Severa & 0 & 0 & 0 & 0 & 0 & 0 & 1 & 11,1 \\
Profunda & 0 & 0 & 0 & 0 & 0 & 0 & 0 & 0 \\
Total & 20 & 100 & 20 & 100 & 9 & 100 & 9 & 100 \\
\hline
\end{tabular}

$\mathrm{OD}$, Orelha direita; $\mathrm{OE}$, Orelha esquerda. 
Tabela 3. Classificação das curvas timpanométricas no sexo feminino portador de EM.

\begin{tabular}{|c|c|c|c|c|c|c|c|c|}
\hline \multirow[b]{3}{*}{ Classificação } & \multicolumn{4}{|c|}{ Sexo feminino } & \multicolumn{4}{|c|}{ Sexo masculino } \\
\hline & \multicolumn{2}{|c|}{ OD } & \multicolumn{2}{|c|}{$\mathrm{OE}$} & \multicolumn{2}{|c|}{ OD } & \multicolumn{2}{|c|}{$\mathrm{OE}$} \\
\hline & $n^{\circ}$ & $\%$ & $n^{\circ}$ & $\%$ & $n^{\circ}$ & $\%$ & $n^{\circ}$ & $\%$ \\
\hline$A$ & 20 & 100 & 18 & 90 & 9 & 100 & 9 & 100 \\
\hline B & 0 & 0 & 1 & 5 & 0 & 0 & 0 & 0 \\
\hline C & 0 & 0 & 0 & 0 & 0 & 0 & 0 & 0 \\
\hline $\begin{array}{l}\text { Perfuração } \\
\text { timpânica }\end{array}$ & 0 & 0 & 1 & 5 & 0 & 0 & 0 & 0 \\
\hline Total & 20 & 100 & 20 & 100 & 9 & 100 & 9 & 100 \\
\hline
\end{tabular}

$\mathrm{OD}$, Orelha direita; $\mathrm{OE}$, Orelha esquerda.

Tabela 4. Classificação dos tipos de ondas de acordo com Jerger (1986) em 58 orelhas de 29 portadores de EM.

\begin{tabular}{lcccc}
\hline & \multicolumn{2}{c}{ Sexo feminino } & \multicolumn{2}{c}{ Sexo masculino } \\
\cline { 2 - 5 } & $\mathrm{N}^{\circ}$ & $\%$ & $\mathrm{~N}^{\circ}$ & $\%$ \\
\hline Tipo I & 22 & 55 & 10 & 55 \\
Tipo II & 14 & 35 & 5 & 27,7 \\
Tipo III & 0 & 0 & 0 & 0 \\
Tipo IV & 2 & 5 & 1 & 5,55 \\
Tipo V & 2 & 5 & 2 & 11,11 \\
Total & 40 & 100 & 18 & 100 \\
\hline
\end{tabular}

Tabela 5. Classificação dos PEATE em ambos os sexos.

\begin{tabular}{|c|c|c|c|c|c|c|}
\hline & \multicolumn{2}{|c|}{ Feminino } & \multicolumn{2}{|c|}{ Masculino } & \multicolumn{2}{|c|}{ Total } \\
\hline & $\mathrm{N}^{\circ}$ & $\%$ & $\mathrm{~N}^{\circ}$ & $\%$ & $\mathrm{~N}^{\mathrm{O}}$ & $\%$ \\
\hline Normal & 8 & 40 & 4 & 44 & 12 & 41,37 \\
\hline Alterado & 12 & 60 & 5 & 56 & 17 & 58,62 \\
\hline Total & 20 & 100 & 9 & 100 & 29 & 100 \\
\hline
\end{tabular}

cie de prata, após limpeza local da pele com álcool absoluto e escarificação com pasta abrasiva. Entre a pele e os eletrodos foram colocados pasta condutiva eletrolítica procedendo então a fixação dos mesmos à pele com esparadrapo. Os indivíduos foram orientados a permanecerem durante as aquisições com os olhos fechados, evitando movimentos palpebrais, mandibulares e cervicais.

Os eletrodos negativos foram fixados nos lobos das orelhas (A1 e $A 2)$, o positivo no vértex $(\mathrm{Cz})$ e o eletrodo comum na região frontal ( $\mathrm{Fpz}$ ), localizações estas definidas pelo sistema internacional $10 / 20^{9}$, permitindo registro simultâneo em dois canais. As impedâncias dos eletrodos foram testadas antes do início das aquisições, sempre se mantendo abaixo de 5 Kohms.
Como estímulo acústico foi utilizado 2000 cliques, apresentados por transdutor supraural TDH - 39, de polaridade rarefeita, com duração do clique de 100 ms, com intensidade de $60 \mathrm{~dB}$ em pico equivalente de nível de pressão sonora (pe NPS) acima do limiar psicoacústico de cada indivíduo. A apresentação do estímulo foi monoaural, mascaramento com ruído branco contralateral em intensidade $30 \mathrm{~dB}$ pe NPS abaixo da intensidade do clique.

Foram utilizados filtros de passa alto em $100 \mathrm{~Hz}$ e passa baixo em $2500 \mathrm{~Hz}$, com janela de $12 \mathrm{~ms}$. Os cliques foram apresentados com ritmo e estimulação em 11 por segundo na orelha direita seguido da orelha esquerda.

Para definição dos limites de normalidade dos PEATE foram utilizados os critérios definidos pela American EEG Society ${ }^{10}$, os valores com 2,5 desvios-padrão acima da média encontrada nos padrões de normalidade, estabelecidos em indivíduos do sexo masculino e feminino sem doenças neurológicas ou otorrinolaringológicas determinados no mesmo equipamento utilizado, conferindo um limite de confiança de 99,38\%.

Todos os indivíduos apresentaram diagnóstico de EM clinicamente definida (EMCD) de acordo com os critérios de diagnóstico propostos por Poser ${ }^{11}$ e tiveram suas formas clínicas classificadas segundo Lublin e Reingold ${ }^{12}$.

Para a classificação das ondas PEATE nos indivíduos portadores de EM foi utilizada a proposta por Jerger ${ }^{5}$ :

Tipo I: resposta normal; todas as ondas presentes com latências e intervalos interpicos normais.

Tipo II: prolongamento de intervalo I-V.

Tipo III: degradação da forma das ondas; morfologia pobre; picos de difícil identificação e/ou amplitudes diminuídas; e/ou razões de amplitude anormais.

Tipo IV: desaparecimento das últimas ondas; ausência das últimas ondas, primeiras ondas presentes com latência normais.

Tipo V: resposta extremamente anormal; somente onda I normal. 
Tabela 6. Valores individuais de latências absolutas e intervalos interpicos em indivíduos portadores de EM do sexo feminino com ritmo de estimulação a 11 cliks por segundo.

\begin{tabular}{|c|c|c|c|c|c|c|c|c|c|c|c|}
\hline \multicolumn{6}{|c|}{ Ondas } & \multicolumn{6}{|c|}{ Intervalos Interpicos } \\
\hline \multicolumn{2}{|c|}{1} & \multicolumn{2}{|c|}{ III } & \multicolumn{2}{|c|}{ v } & \multicolumn{2}{|c|}{$I-I I I$} & \multicolumn{2}{|c|}{ III-V } & \multicolumn{2}{|c|}{$\mathrm{I}-\mathrm{V}$} \\
\hline OD & $\mathrm{OE}$ & OD & $\mathrm{OE}$ & OD & $\mathrm{OE}$ & OD & $\mathrm{OE}$ & OD & $\mathrm{OE}$ & OD & $\mathrm{OE}$ \\
\hline 1,632 & 1,776 & 3,888 & 3,936 & 6,480 & 6,456 & 2,256 & 2,160 & 2,592 & 2,496 & 4,848 & 4,656 \\
\hline 1,680 & 1,824 & 3,600 & 3,792 & 5,616 & 5,802 & 1,920 & 1,968 & 2,016 & 1,968 & 3,936 & 3,936 \\
\hline 1,632 & 1,728 & 3,696 & 4,080 & 5,616 & 5,664 & 2,064 & 2,352 & 1,920 & 1,584 & 3,984 & 3,936 \\
\hline 1,872 & 1,920 & 3,840 & 4,080 & 5,808 & 6,432 & 1,968 & 2,160 & 1,968 & 2,352 & 3,936 & 4,512 \\
\hline 1,632 & 1,776 & 3,696 & 4,176 & 5,808 & 6,384 & 2,064 & 2,400 & 2,112 & 2,208 & 4,176 & 4608 \\
\hline 1,488 & 1,488 & - & 3,600 & - & 5,424 & - & 2,112 & - & 1,824 & - & 3,936 \\
\hline 1,632 & 1,488 & 3,888 & 3,840 & 6,192 & 5,904 & 2,256 & 2,352 & 2,064 & 2,064 & 4,320 & 4,416 \\
\hline 1,920 & 1,344 & 3,744 & 3,888 & 5,664 & 6,096 & 1,824 & 2,544 & 1,920 & 2,208 & 3,744 & 4,752 \\
\hline 1,536 & 1,440 & 3,936 & 3,792 & 6,096 & 5,952 & 2,400 & 2,352 & 2,112 & 2,160 & 4,512 & 4,512 \\
\hline 1,728 & - & 4,176 & - & 6,048 & - & 2,448 & - & 1,872 & - & 4,320 & - \\
\hline 2,016 & 1,920 & 3,936 & 3,744 & 5,904 & 5,712 & 1,920 & 1,824 & 1,968 & 1,968 & 3,888 & 3,792 \\
\hline 1,536 & 1,584 & 3,504 & 3,552 & 5,520 & 5,568 & 1,968 & 1,968 & 2,016 & 2,016 & 3,984 & 3,984 \\
\hline 1,680 & 1,728 & 3,744 & 3,888 & 5,808 & 5,808 & 2,064 & 2,160 & 2,064 & 1,920 & 4,128 & 4,080 \\
\hline 1,632 & 1,920 & 3,504 & 3,840 & 5,328 & 5,808 & 1,872 & 1,920 & 1,824 & 1,968 & 3,696 & 3,888 \\
\hline 1,536 & - & 4,176 & - & 6,192 & - & 2,640 & - & 2,016 & - & 4,656 & - \\
\hline 2,112 & 2,112 & 4,368 & 4,176 & - & 5,952 & 2,256 & 2,064 & - & 1,776 & - & 3,840 \\
\hline 1,872 & 2,112 & 3,840 & 4,032 & 5,904 & 6,192 & 1,968 & 1,920 & 2,064 & 2,160 & 4,032 & 4,080 \\
\hline 1,440 & 1,680 & 3,840 & 3,888 & 5,520 & 5,616 & 2,400 & 2,208 & 1,680 & 1,728 & 4,080 & 3,936 \\
\hline 1,680 & 1,872 & 4,128 & 4,272 & 6,144 & 6,228 & 2,448 & 2,400 & 2,016 & 2,016 & 4,464 & 4,416 \\
\hline 1,680 & 1,776 & 3,792 & 3,792 & 5,664 & 5,664 & 2,112 & 2,016 & 1,872 & 1,872 & 3,984 & 3,888 \\
\hline
\end{tabular}

OD,Orelha direita; $\mathrm{OE}$, Orelha esquerda; - , Ausência de resposta.

Os indivíduos portadores de EM foram classificados como normais, à avaliação com os PEATE, quando apresentavam respostas tipo I em ambos os lados e em alterados quando apresentavam algum dos demais tipos na classificação de Jerger ${ }^{5}$ em, pelo menos, um lado.

Os indivíduos concordaram com os termos de esclarecimento e assinaram o termo de consentimento para a realização dos exames propostos.

\section{RESULTADOS}

Dos 29 casos de EM do estudo 58,62 \% apresentavam a forma surto remissiva, as demais incidências das outras formas encontram-se na Tabela 1 .

Oitenta e seis porcento dos pacientes apresentaram limiares auditivos tonais e vocais dentro da nor- malidade e as perdas auditivas nos demais não interferiram na aquisição dos PEATE (Tabelas 2 e 3).

Dos 58 PEATE realizados encontrou-se $55 \%$ classificados como tipo I na classificação de Jerger ${ }^{5}$, em ambos os sexos. Em 35\% das ondas no sexo feminino e em $27,7 \%$ no masculino encontrou-se prolongamento do intervalo interpico I-V, sendo considerados como tipo II. Não encontrou-se ondas classificadas como tipo III e, os tipos IV e V, que representam uma maior anormalidade nos traçados, ocorreram em $10 \%$ no sexo feminino e em $16,66 \%$ no masculino (Tabela 4).

Considerando-se como alterados as avaliações com PEATE dos indivíduos portadores de EM que apresentaram os tipos II, IV ou V em pelo menos 
Tabela 7. Valores individuais de latências absolutas e intervalos interpicos em indivíduos portadores de EM do sexo masculino com ritmo de estimulação a 11 cliks por segundo.

\begin{tabular}{|c|c|c|c|c|c|c|c|c|c|c|c|}
\hline \multicolumn{6}{|c|}{ Ondas } & \multicolumn{6}{|c|}{ Intervalos Interpicos } \\
\hline \multicolumn{2}{|c|}{1} & \multicolumn{2}{|c|}{ III } & \multicolumn{2}{|c|}{ v } & \multicolumn{2}{|c|}{ I-III } & \multicolumn{2}{|c|}{ III-V } & \multicolumn{2}{|c|}{$\mathrm{I}-\mathrm{V}$} \\
\hline OD & $\mathrm{OE}$ & OD & $\mathrm{OE}$ & OD & $\mathrm{OE}$ & OD & $\mathrm{OE}$ & OD & $\mathrm{OE}$ & OD & $\mathrm{OE}$ \\
\hline 1,680 & 1,824 & 3,888 & 3,984 & 5,856 & 6,000 & 2,208 & 2,160 & 1,968 & 2,016 & 4,176 & 4,176 \\
\hline 1,824 & - & 3,936 & - & 6,192 & - & 2,112 & - & 2,256 & - & 4,368 & - \\
\hline 1,632 & 1,632 & 3,840 & 4,032 & 6,144 & 6,480 & 2,208 & 2,400 & 2,304 & 2,448 & 4,512 & 4,848 \\
\hline 1,728 & 1,920 & 4,032 & 4,224 & 5,952 & 5,856 & 2,304 & 2,304 & 1,920 & 1,632 & 4,224 & 3,936 \\
\hline 1,776 & 1,824 & 4,032 & 4,080 & 5,856 & 5,952 & 2,256 & 2,256 & 1,824 & 1,872 & 4,080 & 4,128 \\
\hline 1,872 & 1,968 & 3,936 & 3,984 & 6,144 & 6,144 & 2,064 & 2,016 & 2,208 & 2,160 & 4,272 & 4,176 \\
\hline 1,872 & 1,776 & 4,080 & 4,320 & 5,760 & 6,048 & 2,208 & 2,544 & 1,680 & 1,728 & 3,888 & 4,272 \\
\hline- & 1,920 & - & 3,840 & - & - & - & 1,920 & - & - & - & - \\
\hline 1,536 & 1,680 & 3,840 & 3,600 & 5,568 & 6,288 & 2,304 & 1,920 & 1,728 & 2,688 & 4,032 & 4,608 \\
\hline
\end{tabular}

OD, Orelha direita; OE, Orelha esquerda; -, Ausência de resposta.

um dos lados, encontrou-se $60 \%$ de alterações no sexo feminino e $56 \%$ no masculino, totalizando $58,62 \%$ (Tabela 5). Os valores individuais das latências absolutas e intervalos interpicos dos indivíduos portadores de EM encontram-se nas Tabelas 6 e 7.

\section{DISCUSSÃO}

A realização dos PEATE em doenças desmielinizantes como a EM é indicada para a avaliação do envolvimento do sistema nervoso central. Alterações nos PEATE podem sugerir a presença de lesão, não suspeitada clinicamente. O objetivo do presente estudo foi avaliar a incidência de alterações audiológicas e nos PEATE em indivíduos portadores de esclerose múltipla utilizando-se da classificação de Jerger ${ }^{5}$ dos PEATE.

No Brasil, Lana-Peixoto e Lana-Peixoto ${ }^{13}$ encontraram sinais e sintomas de alterações de tronco encefálico em $34 \%$ dos pacientes portadores de EM na fase inicial e em $45 \%$ na fase de evolução da doença. Tilbery, Felipe, Baldauf e Peres ${ }^{14}$ encontraram sinais e sintomas de alterações de tronco encefálico em $32 \%$ dos pacientes na fase inicial e em $52 \%$ dos pacientes na fase evolutiva na EM. Em nossa casuística, foram estudados 29 portadores de EM que não apresentavam sinais e sintomas de alterações de tronco encefálico no momento da realização dos PEATE, encontrando-se $58,62 \%$ de individuos com alterações. Papais-Alvarenga e col. ${ }^{15}$ encontraram em 88 portadores de EM uma prevalência do sexo feminino de $76,1 \%$. Nossa casuística foi de $68,96 \%$ de pacientes do sexo feminino e $31,03 \%$ do masculino, confirmando uma maior prevalência do sexo feminino na doença.

Nos resultados dos PEATE de 18 orelhas no sexo masculino e de 40 orelhas no feminino, encontraram-se alterações em $44 \%$ no sexo masculino e em $45 \%$ no feminino, com ritmo de estimulação rotineiramente utilizado em 11 cliques por segundo. Nestes a alteração mais comumente encontrada foi a Tipo II, segundo Jerger ${ }^{5}$, ocorrendo em $27,77 \%$ no sexo masculino e em $35 \%$ no feminino.

No sexo masculino, foram encontrados $56 \%$ de portadores de EM considerados como alterados após a realização dos PEATE e $60 \%$ no feminino, utilizando-se ritmo de estimulação em 11 cliques por segundo. Robinson e Rudge ${ }^{16}$, utilizando estimulação binaural em 20 cliques por segundo, encontraram respostas anormais em $73 \%$ de uma casuística de 30 portadores de EM. Rudge e Robinson ${ }^{17}$ encontraram $51 \%$ de alterações nos PEATE em portadores de EM sem sinais de envolvimento do tronco encefálico, utilizando ritmos de estimulação em 2,5 e 20 cliques por segundo. Kjaer $^{18}$ encontrou $83 \%$ de traçados anormais em um grupo de portadores de EM utilizando ritmo de estimulação em 4 cliques por segundo.

\section{CONCLUSÕES}

A análise de 29 pacientes portadores de EM clinicamente definida mostrou aproximadamente $60 \%$ de indivíduos com alterações nos PEATE. Apesar no número de indivíduos desta casuística, estes acha- 


\section{dos enfatizam a relevância do estudo dos PEATE em casos de suspeita clínica de doenças desmielinizantes e naqueles com diagnóstico definido de EM, mes- mo que não apresentem sinais de sintomas de aco- metimento do tronco encefálico pela doença.}

\section{REFERÊNCIAS}

1. Chiappa KH. Evoked potentials in clinical medicine. 3.Ed. Philadelphia: Lippincott-Raven, 1997:1-30.

2. Hood L. Clinical applications of the auditory brain response. San Diego: Singular Publishing Group.1998:67-91.

3. KurtzkeJF, Beebe GW, Dagler B, Auth TL, Kurland LT, Defzger MD. Studies on the natural history of multiple sclerosis: clinical and laboratorial findings at first diagnoses. Acta Neurol Scand 1972;48:19-46.

4. McDonald WB. Diagnosis of multiple sclerosis. BMJ1989;299:635-637.

5. Jerger JF, Oliver TA, Chmiel RA, Rivera VM. Patterns of auditory abnormality in multiple sclerosis. Audiology 1986;25:193-209.

6. Mangabeira Albernaz P, Mangabeira Albernaz PL, Mangabeira Albernaz LG, Mangabeira Albernaz $\mathrm{F}^{\circ} \mathrm{P}$. Exame da função auditiva. In Otorrinolaringologia Prática. São Paulo: Sarvier, 1973:40.

7. Davis H, Silverman SR. Hearing and Deafness. Copyright, 1970.
8. Russo ICP, Achados imitanciométricos em pré-escolares de níveis sócio econômico baixo, médio ealto: estudo comparativo. Acta AWHO1988;4:229-235.

9. Jasper $\mathrm{HH}$. The ten twenty eletrode system of the international federation. Electroencephalo Clin Neurophysiol 1958;10:371-375.

10. American EEG Society. Clinical evoked potentials guidelines: recomended standarts for normative studies of evoked potentials, statistical analyses of results and criteria for clinically significant abnormality. J Clin Neurophysiol 1994;11:45-47.

11. Poser CM, Paty DW, Scheinberg L, et al. New diagnostic criteria for multiple sclerosis: guidelines for research protocols. Ann Neurol 1983;13:227-231.

12. Lublin FD, Reingold SC. Defining the clinical course of multiple sclerosis: results of an international survey. Neurology 1996;46:907-911.

13. Lana-Peixoto M A, Lana-Peixoto M I V. Is multiple sclerosis in Brazil and Asia alike? Arq Neuropsquiatr 1992;50:419-425.

14. Tilbery C P, Felipe E, Baldauf CM, Peres M F P. Esclerose múltipla: análise clínica e evolutiva de 214 casos. Arq Neuropsquiatr 1995;53:203-207.

15. Papais-Alvarenga RM, Santos C M M, Abreu J S, et al. Esclerose múltipla : perfil clínico e evolutivo no município do Rio de Janeiro. Rev Bras Neurol 1995;31:75-87.

16. Robinson K, Rudge P. Auditory evoked responses in multiple sclerosis. Lancet 1975;24:1164-1166.

17. Rudge $\mathrm{P}$, Robinson K. Abnormaties of the auditory evoked potentials in patients with multiple sclerosis. Brain 1977;100:19-40.

18. Kjaer M. Variations of brain stem auditory evoked potentials correlated to duration and severity of multiple sclerosis. Acta Neurol Scand 1980;61:157-166. 